\title{
El celecoxib podría ser más seguro que otros AINE en pacientes con insuficiencia cardiaca congestiva
}

Hudson M et al. BMJ 2005;330:1370.

\section{Objetivo}

Comparar el riesgo de muerte y de recurrencia de insuficiencia cardiaca en pacientes añosos (edad mayor o igual a 66 años) tratados con celecoxib, rofecoxib u otros antiinflamatorios no esteroideos (AINE).

Diseño

Estudio de cohorte retrospectiva.

Lugar

Canadá (registros hospitalarios de Québec).

\section{Pacientes}

Portadores de insuficiencia cardíaca congestiva, 717 expuestos a celecoxib, 869 a rofecoxib y 280 a otros AINE.

\section{Evaluación de los factores de riesgo}

La exposición a fármacos se documentó a través de los registros de la base de datos de la cohorte. Tales registros fueron validados. El ajuste multivariado* tuvo en cuenta confundidores* ${ }^{*}$ potenciales tales como edad, sexo, comorbilidades, otras prescripciones, características del médico o del centro tratante, infarto de miocardio en los tres años previos, tiempo a la primera prescripción y episodios de insuficiencia cardíaca congestiva previos a la primera prescripción.

\section{Medición de resultados principales}

1) Resultado primario combinado: tiempo hasta la muerte o hasta la recurrencia. 2) Resultados secundarios: tiempo hasta la muerte o tiempo a la recurrencia.

\section{Resultados principales}

El riesgo de muerte e insuficiencia cardíaca recurrente fue mayor en los pacientes que recibieron AINE o rofecoxib que en los que recibieron celecoxib (RR 1.26 y 1,27 respectivamente). En la tabla se detallan los principales hallazgos, tanto del punto final combinado como de cada uno de ellos por separado.

Tabla. Resultados principales

\begin{tabular}{|c|c|c|}
\hline & Riesgo Relativo & IC95\% \\
\hline \multicolumn{3}{|c|}{ Muette o Recurrencia } \\
\hline Otros AINE vs celecoxib & 1.26 & 1.00 a 1.57 \\
\hline Rofecoxib vs celecoxib & 1.27 & 1.09 a 1.49 \\
\hline Otros AINE vs rofecoxib & 0.99 & 0.80 a 1.22 \\
\hline \multicolumn{3}{|c|}{ Muerte } \\
\hline Otros AINE vs celecoxib & 1.54 & 1.17 a 2.04 \\
\hline Rofecoxib vs celecoxib & 1.44 & 1.17 a 1.78 \\
\hline Otros AlNE vs rofecoxib & 1.07 & 0.82 a 1.39 \\
\hline \multicolumn{3}{|c|}{ Recurrencia } \\
\hline Otros AINE vs celecoxib & 1.21 & 0.92 a 1.60 \\
\hline Rofecoxib us celecoxib & 1.17 & 0.96 a 1.42 \\
\hline Otros AINE vs rofecoxib & 1.04 & 0.80 a 1.36 \\
\hline
\end{tabular}

\section{Conclusiones}

El celecoxib pareció más seguro que el rofecoxib y que otros AINE en pacientes mayores de 65 años con insuficiencia cardíaca congestiva.

\section{Comentario}

Los potenciales riesgos cardiovasculares, específicamente, la posible asociación entre el empleo de ciertos AINE (especialmente el rofecoxib) y el desarrollo de eventos coronarios se halla actualmente en el foco de la investigación farmacoepidemiológica ${ }^{1,2,3}$. Pero, además, siempre ha sido objeto de interés el estudio de la seguridad de los AINE en pacientes con insuficiencia cardíaca congestiva, especialmente aquellos de edad avanzada, dados los múltiples mecanismos a través de los cuales tales agentes podrían resultar dañinos en estos sujetos ${ }^{4}$. En este trabajo, los autores encuentran un menor riesgo de muerte y recurrencia de episodios de insuficiencia cardíaca en los tratados con celecoxib, frente a los que recibieron rofecoxib o aún otros AINE. Los estudios de diseño tipo cohorte retrospectiva están sujetos a sesgos de selección ${ }^{*}$ y de información* (por ejemplo, los pacientes pudieron estar tomando otros AINE no registrados; no siempre es posible controlar la adherencia al tratamiento; etc), y, además, resulta en ellos difícil evaluar el papel de confundidores no correctamente registrados en la historia de los pacientes. Ahora bien; teniendo todo esto en cuenta, ¿podemos concluir, como parecen sugerir los autores, diferencias entre rofecoxib y celecoxib en lo que respecta a los resultados considerados?. No nos parece suficiente la información obtenida como para concluir una diferencia intraclase entre los distintos AINE COX 2 selectivos. Además de las limitaciones propias del diseño, los autores no brindan sustento en términos de plausibilidad biológica que explique las diferencias sugeridas.

\section{Conclusión del comentador}

El empleo de AINE podría estar asociado a eventos adversos, recurrencia y aún muerte en pacientes con insuficiencia cardiaca. Sin embargo, poca es la información que agrega este trabajo respecto a las diferencias entre agentes sobre el particular, y no puede sostenerse aún, ni desde el diseño empleado ni desde lo actualmente conocido en términos de plausibilidad biológica, una diferencia intraclase entre los subtipos de inhibidores selectivos COX 2. De todos modos, el tema queda planteado y es posible que despierte el interés en términos de desarrollo de futuros estudios comparativos en este terreno.

Claudio Gonzalez [ Depto de Farmacología, UBA. Cátedra de Farmacología, Instituto Universitario CEMIC. ]

Gonzalez C. El celecoxib podría ser más seguro que otros AINE en pacientes con insuficiencia cardíaca congestiva. Evid. act. pract. ambul 9(1):8. En-Febr.2006. Comentado de: Hudson, M., Richard, H., Pilote, L. Differences in outcomes of patients with congestive heart failure prescribed celecoxib, rofecoxib, or non-steroideal anti-inflammatoru drugs: population based study. BMJ 2005; 330:1370. PMID 15947399

\section{Referencias}

1. Ray, W., Stein, M., Hall, K. and col. Non-steroidal anti-inflammatory drugs and risk of serious coronary heart disease: observational cohort study. Lancet 2002; 359: $118-123$

2. Juni, P., Nartey, L., Reichenback, S. and col. Risk of cardiovascular events and rofecoxib: cumulative meta-analysis. Lancet 2004; 364: 2021-2029

3. Couzin J. Drug Safety. Withdrawal of Vioxx cast a shadow over COX-2 inhibitors. Science 2004; 306:384-385

4. García Rodríguez, LA, Hernández-Díaz, S. Nonsteroidal antiinflamatory drugs as a trigger of clinical heart failure. Epidemiology 2003; 14:240-246 